\title{
Dermatologenexlibris der Gegenwart
}

\section{Bookplates of Contemporary Dermatologists}

\section{Zusammenfassung}

Nach kurzer Darstellung der Geschichte und des Gebrauchs von Exlibris werden heute von Dermatologen genutzte Buchmarken gezeigt und besprochen.

\section{Abstract}

History and use of book-plates are described and some representative book-plates of dermatologists used in our time are demonstrated.
In den frühen Jahren des 20. Jahrhunderts war der Besitz und Gebrauch eines Exlibris für jeden, der mehr als zwei Bücher besaß, sozusagen selbstverständlich. Seit dem Zweiten Weltkrieg ist jedoch diese Art, sein Bucheigentum zu kennzeichnen, zunehmend verlassen worden. Manche jüngere Zeitgenossen wissen nicht mehr, was ein Exlibris ist, und im Zeitalter von Paperback und Internet ist auch die Frage berechtigt, ob für seine Verwendung noch eine Notwendigkeit besteht. Es sind daher wohl einleitend einige allgemeine Vorbemerkungen über Zweck und Anwendung von Exlibris erforderlich. Sie sollen verdeutlichen, dass es sich bei älteren Buchmarken um kulturhistorisch und buchgeschichtlich bedeutsame Dokumente handelt, bei neueren Exlibris guter Qualität oft um kleine Kunstwerke, die zur Beschäftigung mit ihren Schöpfern anregen können und immer auch zu Nachforschungen über ihre Eigner.

Exlibris wurden und werden seit etwa fünfhundert Jahren verwendet. In dieser Zeit hat sich ihre Gestaltung, aber auch die Motivation ihrer Benutzer zeitabhängig verändert. Als gegen Ende des 14. Jahrhunderts Institutionen oder Personen, die eine größere Zahl von Büchern in Besitz hatten, damit begannen, diese durch ein fest eingeklebtes kleines Blatt mit ihrem Namen oder ihrem Wappen als ihr Eigentum zu kennzeichnen, war dies zunächst durch den hohen materiellen Wert der Bücher veranlasst.
Auch nach der Erfindung des Drucks mit beweglichen Lettern waren Druck und Herstellung von Büchern noch über Jahrhunderte so teuer, dass eine größere Sammlung von Büchern nur für Fürstenhäuser, reiche Kirchen und Klöster, Hochschulen und wenige wohlhabende Kaufmannsfamilien oder Gelehrte erschwinglich war. Damit hatte Buchbesitz einen hohen Prestigewert, was auch verständlich macht, dass die Eigner schon bald auf eine gute grafische Gestaltung ihrer Exlibris Wert legten. Frühe Exlibris sind u. a. von Albrecht Dürer, Bartel Beham, Lucas Cranach und Hans Holbein angefertigt worden. Zu ihren Eignern zählten schon seit etwa 1500 immer auch Ärzte.

Weiteste Verbreitung und ihren qualitativen Höhepunkt fanden Exlibris gegen Ende des 19. und im frühen 20. Jahrhundert, nachdem insbesondere die Künstler des Jugendstils das Exlibris als spezielles künstlerisches Ausdrucksmittel entdeckten. Obwohl zu dieser Zeit Bücher für jeden zugänglich geworden waren, hatten viele Eigner den Wunsch, die ihnen besonders wertvollen Bücher mit ihrer persönlichen Buchmarke zu kennzeichnen. Für diese Bücher wünschte man sich auch kostbare Exlibris, die dem Buch einen zusätzlichen Wert geben sollten. Neben Max Klinger, Heinrich Vogeler, Otto Ubbelohde und Emil Orlik, die die Gestaltung und Entwicklung der Exlibris in Deutschland besonders geprägt haben, haben auch fast alle anderen bedeutenden Künstler

Institutsangaben

Dermatologische Klinik der Universität des Saarlandes, Homburg

Korrespondenzadresse

Prof. Dr. Hansotto Zaun - Universitätsklinikum des Saarlandes - Klinik für Dermatologie, Allergologie,

Umweltmedizin·66421 Homburg/Saar · E-mail: Zaun-Homburg@t-online.de

Bibliografie

Akt Dermatol 2004; 30: 256-262 @ Georg Thieme Verlag Stuttgart · New York .

DOI 10.1055/s-2004-814442 · ISSN 0340-2541 
der ersten 40 Jahre des 20. Jahrhunderts gelegentlich Exlibris geschaffen, zuweilen nur für ihre Angehörigen und ihre Freunde oder auch für ihre Ärzte (so z. B. Hans Thoma für seinen Hausarzt und Förderer Dr. Otto Eiser, Heinrich Zille für seinen Hausarzt und Freund Dr. Adolf Heilborn).

Im Idealfall ist ein Exlibris das Ergebnis der künstlerisch gekonnten Umsetzung von Motivvorstellungen, die vom Eigner vorgegeben oder gemeinsam mit dem Gestalter entwickelt wurden. Der Briefwechsel zwischen unserem Kieler Fachkollegen Carl Schirren (1861 - 1921) und Max Klinger, den der Enkel von C. Schirren herausgegeben hat [8], zeigt eine solche „Kooperation“, die das Exlibris für den Eigner zu einem ganz persönlichen Kunstwerk macht.

Über Bücherzeichen für Ärzte und die darauf dargestellten Motive gibt es eine umfangreiche Literatur z. B. $[2,4,6,14,15,17]$. Besondere Verdienste um das Entdecken, Sammeln und Dokumentieren von Dermatologenexlibris hat sich Albrecht Scholz erworben [9-13], der auch mehrfach auf Tagungen Exlibris für Hautärzte ausgestellt hat. In seinem schönen Buch zu diesem Thema [10] sind Exlibris vor und um 1900 sowie neuere Exlibris bis etwa 1990 von Dermatologen verschiedener Länder besprochen und gezeigt (ca. $80 \mathrm{Abb}$.), darunter viele Blätter berühmter Dermatologen.

Nachfolgend sollen beispielhaft einige Dermatologenexlibris aus unserer Zeit und ihre Eigner vorgestellt werden. Heutige Exlibriseigner sind zumeist Sammler von (z.B.) kostbaren Büchern, seltenen Musiknoten, künstlerischer Mappengrafik und/oder Kleingrafik, die mit ihren Exlibris ihre Sammlung als etwas ihnen besonders Wichtiges auszeichnen wollen. Viele von ihnen haben mehrere Exlibris auf ihren Namen mit oft ganz unterschiedlichen Motiven, die sich auf Beruf, Liebhabereien, Sammelgebiete oder andere Themen beziehen können, häufig auch deshalb mehrere Exlibris, weil anspruchsvolle Drucktechniken wie Kupferstich, Radierung oder Holzschnitt nur eine begrenzte Anzahl von Abzügen erlauben.

Zwei Exlibris des früheren Direktors der Fachklinik Hornheide/ Münster, Franz Ehring, sind vermutlich zahlreichen Kollegen bekannt, da sie mehrfach in der Literatur und in Ausstellungen gezeigt wurden (siehe z. B. $[6,10,17])$. Auf einem, von unserem Kollegen Dietrich Janke gezeichnet, sitzt Ehring auf dem Dach seiner Klinik und wehrt einen Wolf (Lupus), einen Krebs und einen kleinen Pilz ab. Wolf und Krebs finden sich auch auf dem zweiten von Marianne Schroer geschaffenen Blatt.

1991 hat der Maler Wynand Janssens für Franz Ehring ein weiteres Exlibris (Abb.1) geschaffen, das in sehr eindrucksvoller Weise das besondere Verhältnis des Eigners zu alten Büchern darstellt. Ehring schreibt dazu: ,... seit 1950 sammle ich alte Bücher, vor allem aus der Dermatologie. Erst ... im Ruhestand fand ich die Muße, sie zu studieren... Janssens hat dies mit einem aufgeschlagenen Buch dargestellt.

Der Text besagt, dass „die Kenntnis der Historie es erleichtert, gegenwärtige Schwierigkeiten zu bewältigen“ [3]. Vorlagen für die Schriften waren Buchstaben aus Schriften des Paracelsus, 1605 in Straßburg gedruckt (deutsche Frakturschrift) sowie einem 1535

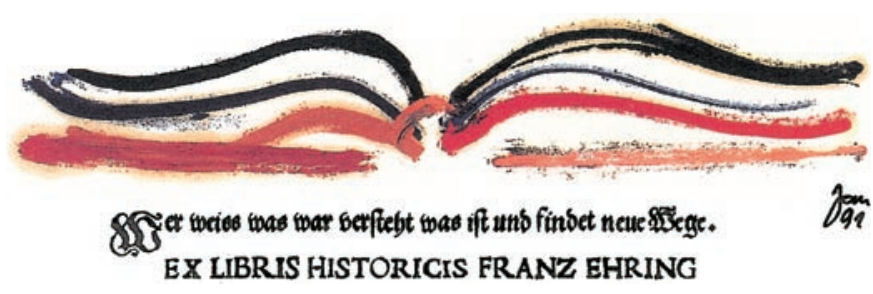

Abb. 1

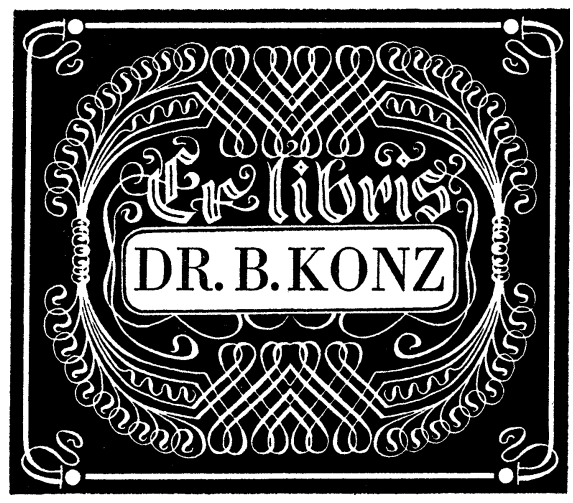

Abb. 2

in Venedig gedruckten Werk des Aristoteles (lateinische Antiqua), beides Druckwerke vom Beginn der Neuzeit.

Birger Konz, langjähriger Leiter der operativen Abteilung der Hautklinik der LMU und maßgeblich prägender Mitgestalter der Vereinigung operative Dermatologie, verwendet für einen Teil seiner Bücher einen Holzschnitt von Rudolf Rieß, der nur den Eignernamen in kunstvoller Umrahmung zeigt (Abb.2), daneben ein jugenstilhaftes Blatt von Christa Weissenfeld [10].

Jan Hrkal, niedergelassener Hautarzt in Neunkirchen/Saar, beschäftigt sich seit seiner Jugend mit Malen und hat einen ganz eigenen Malstil mit kräftigen farbigen Strukturen und unter Verwendung von sehr viel Gold entwickelt. Mehrfach hat er im Saarland sowie in Mannheim und Berlin ausgestellt. Nach Studium und Fachausbildung in Prag kam er 1984 in die Bundesrepublik, hat längere Zeit an meiner Klinik gearbeitet, „nebenbei“ für mich ein von mir gern genutztes Exlibris gezeichnet. Hrkal hat eine größere Sammlung tschechischer Exlibris hoher Qualität und auf seinen Namen mehrere von Prager Künstlerinnen geschaffene Blätter. Eine Radierung von Helena Chroustova zeigt einen Baum, um den sich wie um einen Äskulapstab eine Schlange windet (Abb. 3).

Albrecht Scholz hat an der Dresdener Medizinischen Akademie „Carl Gustav Carus“ unter Heinz Egon Kleine-Natrop seine fachärztliche Weiterbildung absolviert und sich 1981 habilitiert. Sein fachlicher Schwerpunkt war die operative Dermatologie.

Aufgrund besonderen Interesses an grafischer Kunst sammelt er seit vielen Jahren grafische Blätter mit medizinischer Thematik und Ärzteexlibis. Letztere interessieren ihn als Dokumente, die uns gleichermaßen personenbezogene wie auch kunstgeschichtliche und zeitgeschichtliche Informationen vermitteln können. 


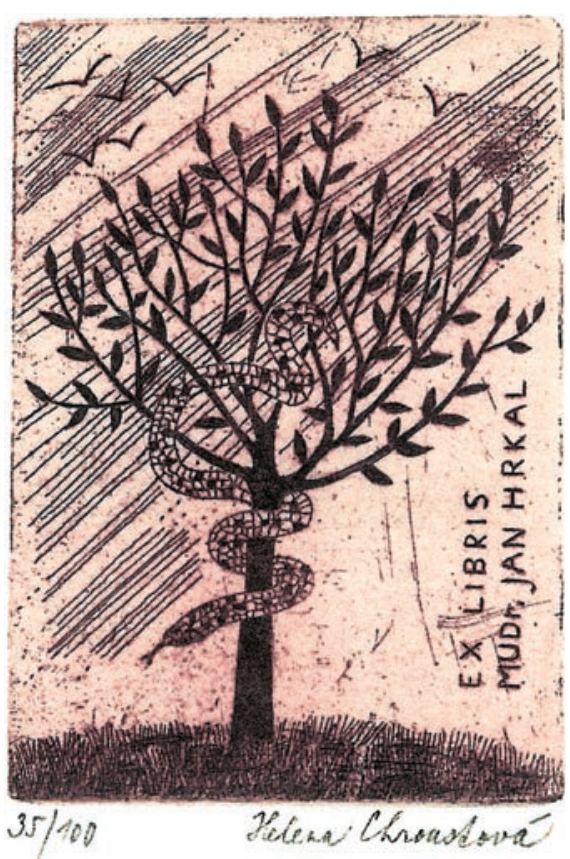

Abb. 3

Abb. 4

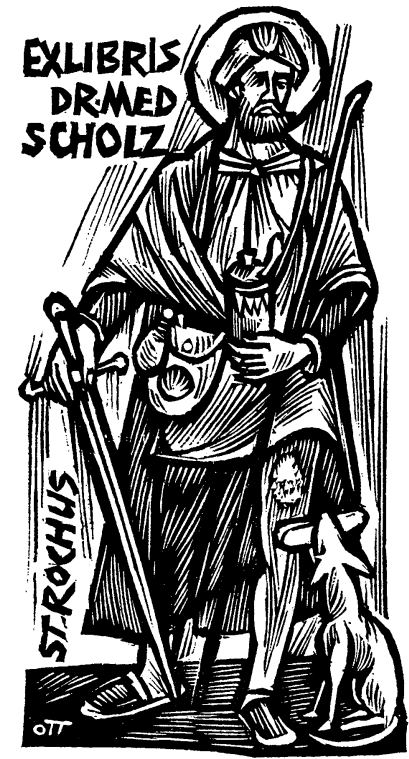

6.

Die glückliche Verbindung von medizingeschichtlichen und kunstgeschichtlichen Interessen hat dazu geführt, dass er heute Direktor des Instituts für Geschichte der Medizin der Dresdener Fakultät ist. In dieser Funktion hat er für sein Institut eine umfangreiche Sammlung von Kunstwerken zum Thema „Arzt, Patient und Krankheit in der Kunst" angelegt und viel beachtete Ausstellungen veranstaltet.

Scholz hat sehr zahlreiche und nach Thematik und Motiven vielgestaltige Exlibris für sich selbst fertigen lassen. Zwei „dermatologische“ Motive zeigen die Abb. 4 und 5: Herbert Ott, ein unter Exlibrisfreunden sehr geschätzter Künstler, hat einen St. Rochus in Holz geschnitten, Werner Schinko eine sich häutende Schlange gezeichnet.

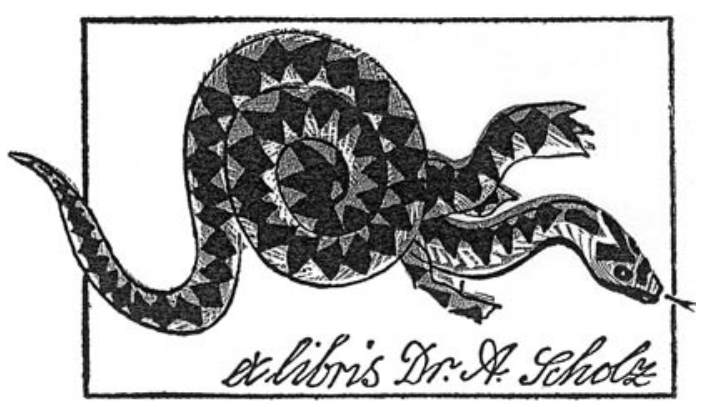

Abb. 5

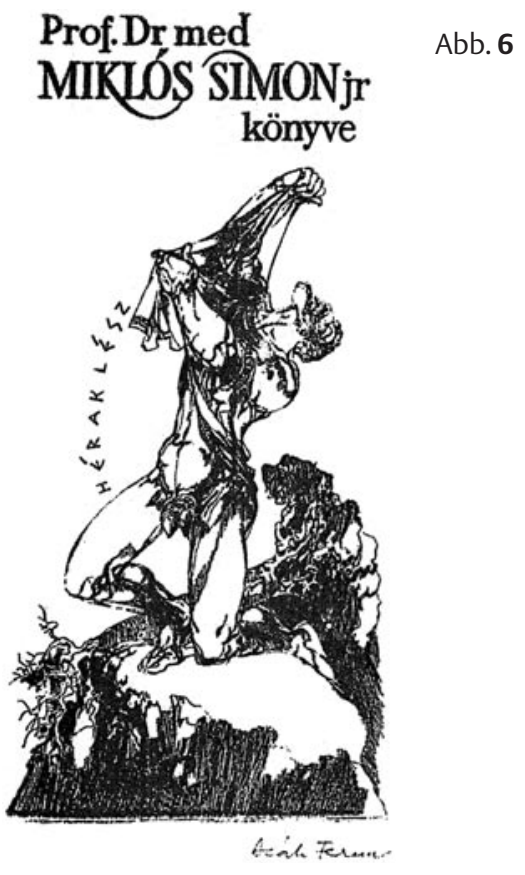

Ein sich häutendes Subjekt zeigt auch das Exlibris von Miklos Simon jr., dem leitenden Oberarzt der Dermatologischen Universitätsklinik Erlangen. Die Zeichnung des ungarischen Künstlers Ferenc Deak (Abb. 6) zeigt Herakles, wie er sich die unerträglich brennende Haut abreißt, die durch den Mantel der Deianeira vergiftetet wurde.

Ernst G. Jung, vormaliger Direktor der Hautklinik Mannheim und Herausgeber dieser Zeitschrift, erhielt zu seinem 60. Geburtstag von den ärztlichen Mitarbeitern seiner Klinik (und auf Anraten seiner Frau Lilian) ein Exlibris, das vielfältige Beziehungen zu Person und Beruf des Eigners erkennen lässt. Die Darstellung des Bremer Malers Heinrich Honkomp (Abb. 7) zeigt hinter einem geöffneten Vorhang und unter einem wehenden Wimpel (Erfolgsymbol) eine strahlende Sonne, die auf zwei Personen herabscheint. Diese Personengruppe erlaubt einerseits die Deutung, dass der Arzt dem Patienten den „kranken Mantel“ seiner entzündlich geröteten Haut mittels Sonne (Fototherapie) abstreift, andererseits kann darin gesehen werden, dass der Arzt durch Anlegen des Mantels (Lichtschutz) den Patienten vor Schaden bewahrt. Lichtschutz und Lichttherapie waren Schwerpunkte der Arbeit von Jung. Dass beides dem ärztlichen Auftrag entspricht zeigt die Äskulapschlange auf dem rechtsseitigen Stein. Das Motto „Die Strahlen der Sonne vertreiben die Nacht“ ist Mozarts Zauberflöte entnommen, die Jung als Kunstwerk und als „maurerisches Bekenntnis“ hoch schätzt. Auf seinem Exlibris empfindet 


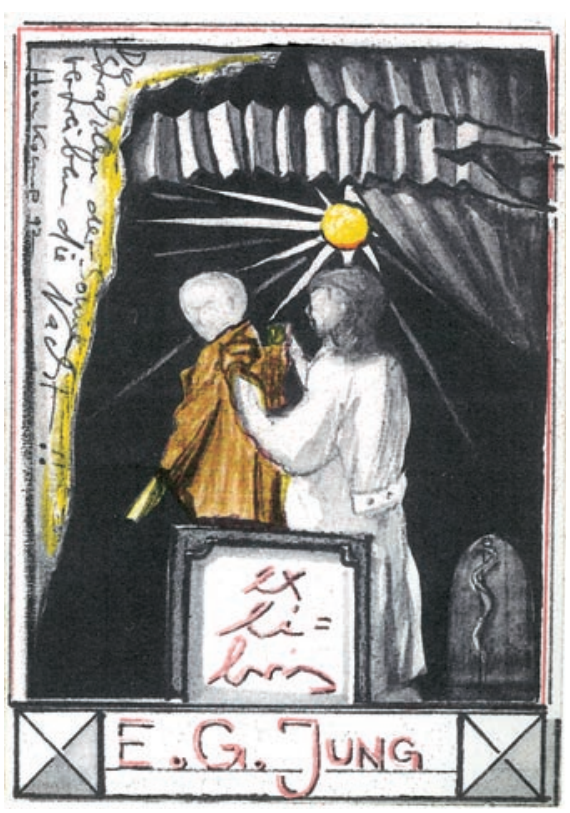

Abb. 7

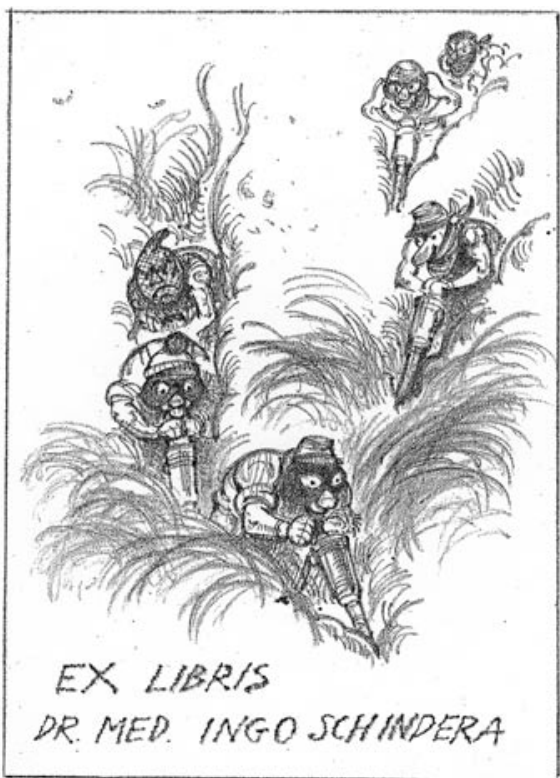

Abb. 8

er dieses Motto auch als ehrende Erinnerung an die vielen Vorfahren sowohl von ihm als auch von seiner Frau Lilian, die in der Freimaurerei segensreich wirkten [5].

Ingo Schindera ist niedergelassener Dermatologe in Völklingen und war langjährig Landesgruppenleiter des Berufsverbandes Deutscher Dermatologen. Sein hier gezeigtes Exlibris, das der Prager Künstler Karel Thoman geschaffen hat (Abb.8), erlaubt gleichfalls zwei Deutungen. Zum einen erkennt man darin die Milben, die mit Eifer ihre Gänge durch die Haut bohren, zum anderen die Kumpels, die in bzw. um Völklingen verschmutzt und mit schweren Hämmern die Kohle aus den Gruben bohren. Im Namen des Berufsverbandes hat mir Ingo Schindera zu meinem 60. Geburtstag eine nur in Kleinstauflage gedruckte Radierung von G. Fiance (Abb.9) geschenkt, die eindrücklich die von den Krabbeltieren auf ihrer Haut verursachten Qualen einer Patientin zeigt.

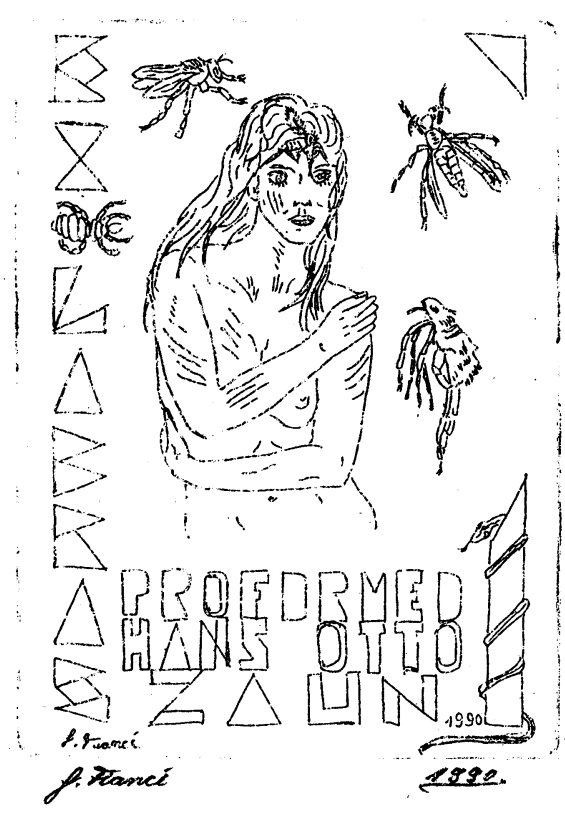

Abb. 9

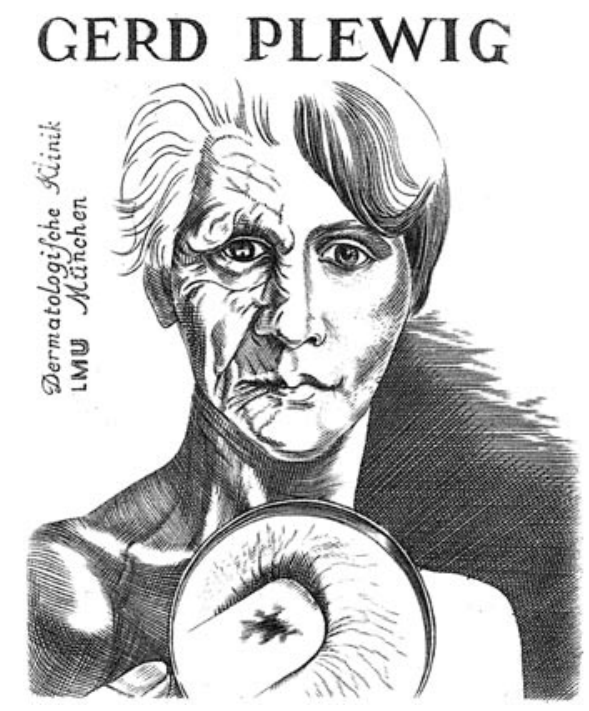

Abb. 10

Für Gerd Plewig, den Direktor der Hautklinik der LMU München, hat Jürgen Czaschka den Wandel der Haut von jugendlicher Glätte und Schönheit zu Falten und Ergrauen im Alter überaus gekonnt dargestellt und nebenbei - unter der Lupe - auf das uns stets begleitende Problem des dunklen Flecks und seiner Deutung angespielt (Abb.10).

Für Heinz-Egon Kleine-Natrop, den vormaligen Direktor der Hautklinik der Medizinischen Akademie Dresden, hat der mit ihm befreundete Meißner Grafiker Lothar Sell eine Serie von Holzschnitten gefertigt, die den Eigner bei verschiedenen hautärztlichen Tätigkeiten zeigt, hier (Abb. 11) beim Nähen einer Exzisionswunde. Detailliertere Angaben über Kleine-Natrop finden sich bei Scholz [10].

Eine Rarität unter den Exlibris für Dermatologen ist das „sprechende Exlibris“ (s.u.) des Direktors der Lübecker Universitätsklinik für Dermatologie, Helmut H. Wolff, der sich wissenschaftlich intensiv mit dermatologischer Elektronenmikroskopie und Histologie befasst hat. Die Künstlerin Marianne Kühnel - Malerin 


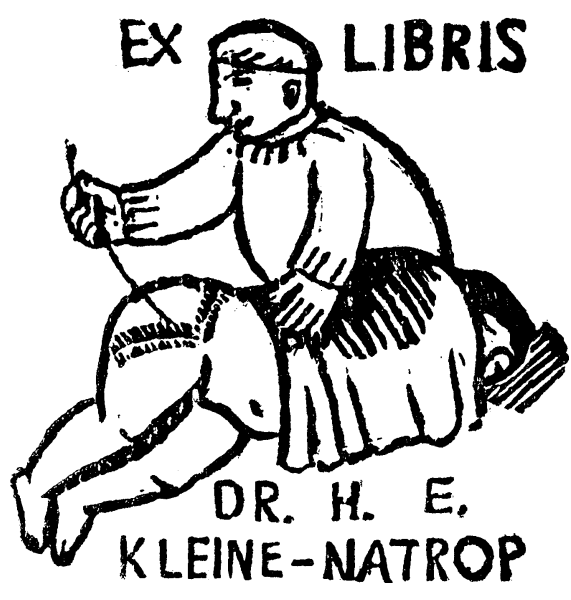

Abb. 11
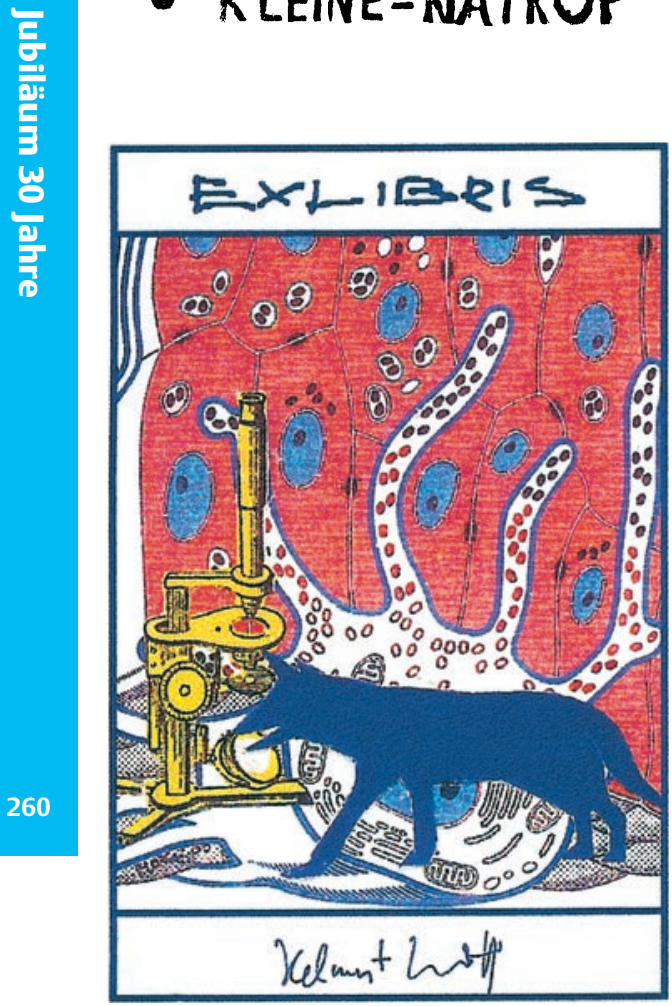

Abb. 12

und Bühnenbildnerin - hat das Blatt wie eine Bühne gestaltet (Abb.12): im Hintergrund eine elektronenmikroskopische Wiedergabe vom Melanozyten und Keratinozyten, links als Kulisse ein historisches Lichtmikroskop, davor als Protagonist der Wolf(f). Der Eigner sagte mir dazu, dass für den Hintergrund wohl der Ehemann der Künstlerin, Wolfgang K. (Prof. emer. für Anatomie und Experte für Elektronenmikroskopie in Lübeck), „ein bisschen Pate gestanden“ habe [16]. Die seltenen Exlibris, auf denen der Name des Eigners, wie hier, bildlich umgesetzt ist, werden als „sprechende Exlibris“ bezeichnet.

Ulrike Schwäblein-Sprafke, die ich viele Male vor der Wende bei Tagungen der Ungarischen Dermatologischen Gesellschaft traf, ist niedergelassene Dermatologin in Hohenstein-Ernstthal. Nach der Wende hat sie sich sehr für den in den östlichen Bundesländern neu begründeten Berufsverband Deutscher Dermatologen eingesetzt. Georg Schindler hat für sie ein Exlibris geschaffen, das neben dem Mikroskop und weiteren minutiös dargestellten Werkzeugen ihrer ärztlichen Tätigkeit auch ihre Vorliebe für grafische Kunst deutlich macht (Abb.13).

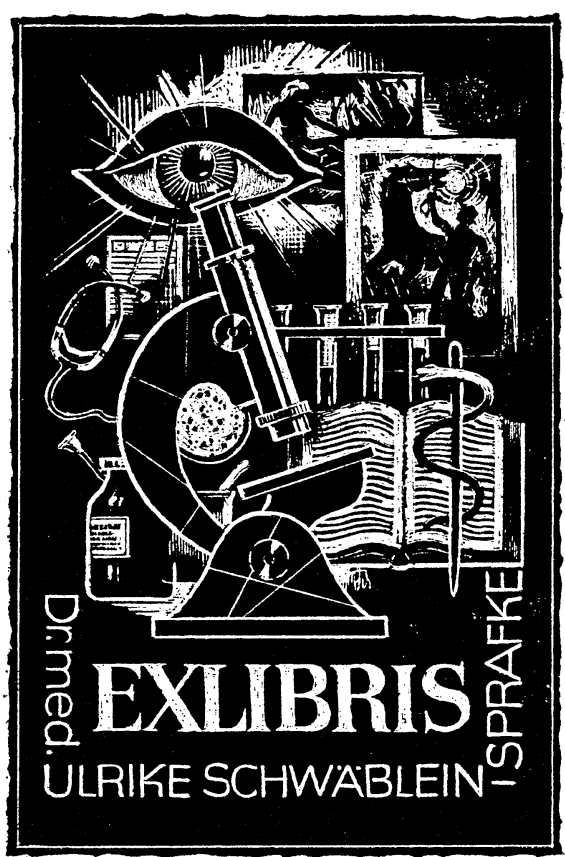

Abb. 13

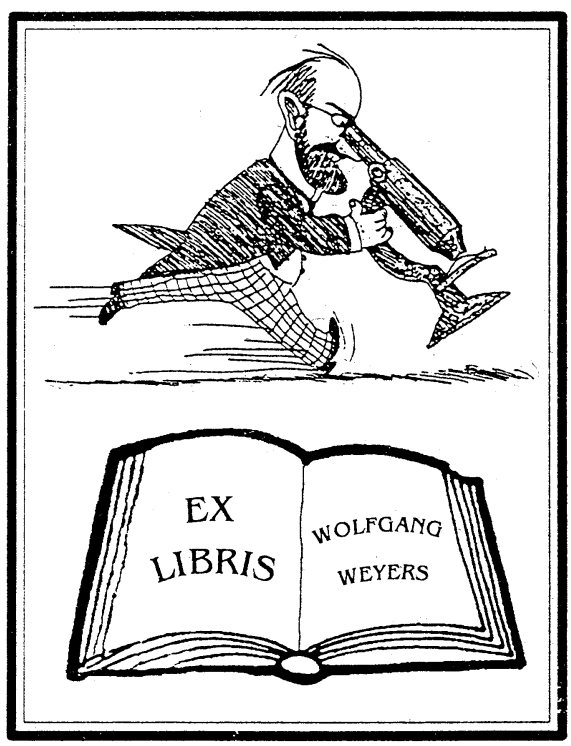

Abb. 14

Ein Mikroskop zeigt auch das heitere Exlibris von Wolfgang Weyers (Abb. 14), das von Hans Traxler gezeichnet wurde. Weyers ist als bekannter Dermatohistologe in Freiburg niedergelassen. $\mathrm{Zu}$ seinen hohen Verdiensten gehört die Abfassung der Monografie „Death of Medicine in Nazi Germany“, in der insbesondere das Schicksal unserer jüdischen Fachkollegen eingehend beschrieben ist.

Wolfgang Peter Herrmann, vormaliger Direktor der Dermatologischen Klinik am Städt. Krankenhaus St. Jürgenstraße in Bremen einer der ältesten großen Hautkliniken Deutschlands - ist ein Freund und Sammler hochwertiger Kunst.

Neben mehreren eigenen Exlibris (siehe z.B. [10]) hat er eine kleinere, aber sehr schöne Exlibrissammlung. Sein hier gezeigtes Exlibris (Abb.15) ist von dem für seine charakteristischen Holz- 

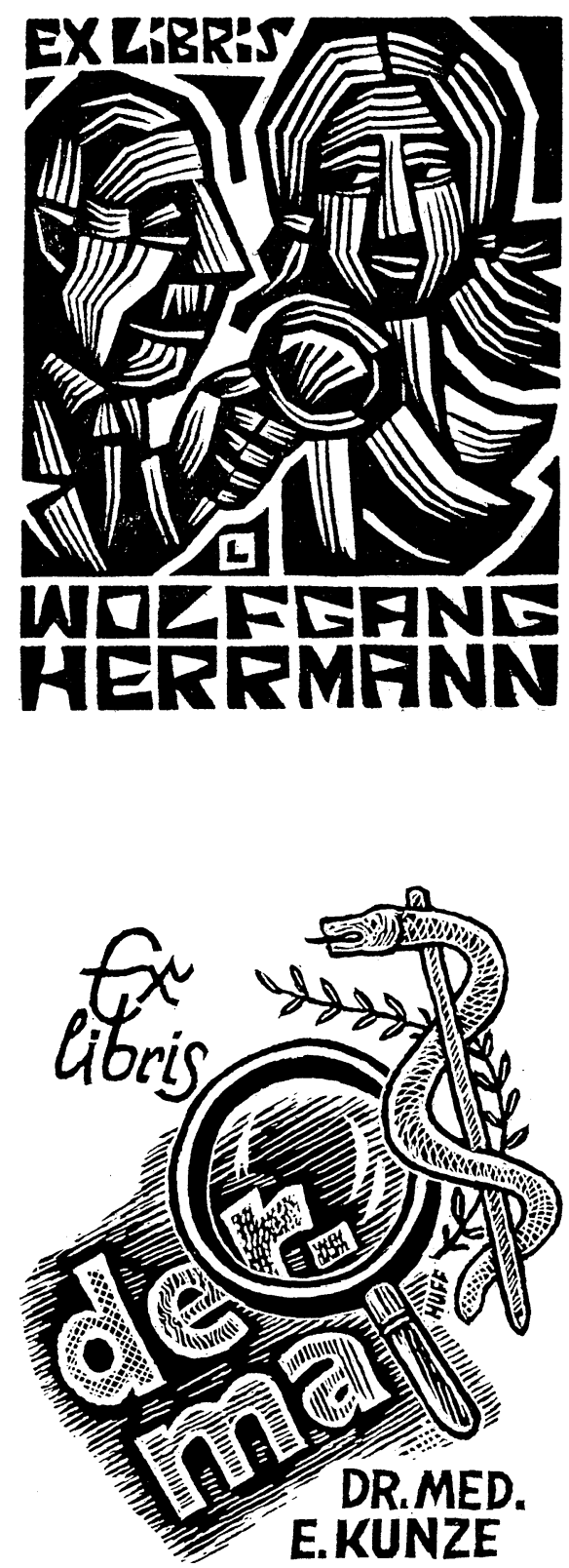

Abb. 16

schnitte bekannten polnischen Künstler Rajmund Lewandowski geschnitten und zeigt den Hautarzt mit seinem unverzichtbaren Hilfsmittel, der Lupe. Die Lupe sehen wir auch auf einem Exlibris für den Hamburger Hautarzt Emil Kunze.

Das in Schabeblatt-Technik gefertigte Exlibris (Abb.16) hat Hermann Huffert geschaffen, der in Sammlerkreisen für seine meist heiter gestimmten Buchmarken bekannt ist. Kunze gehört $\mathrm{zu}$ den Sammlern, die den Fortbestand der Exlibris-Kultur mit großer Begeisterung fördern und durch Aufträge an zahlreiche Exlibriskünstler stützen. Er besitzt über einhundert verschiedene Exlibris auf seinen Namen mit unterschiedlichsten Motiven.

Zum Thema AIDS hat Utz Benkel in Linolschnitt-Technik eine Mappe mit zehn Exlibris [1] für ihn geschaffen, aus der ich ein Blatt zeigen möchte (Abb.17). Kunze schreibt dazu: „Das versinnbildlichte Sprichwort „An jedem Finger eine“ wird dargestellt vor der Kulisse eines imaginären Theatre de la vie, das das Stück „AIDS“ in seinem Programm hat. Provozieren in der Bildge-

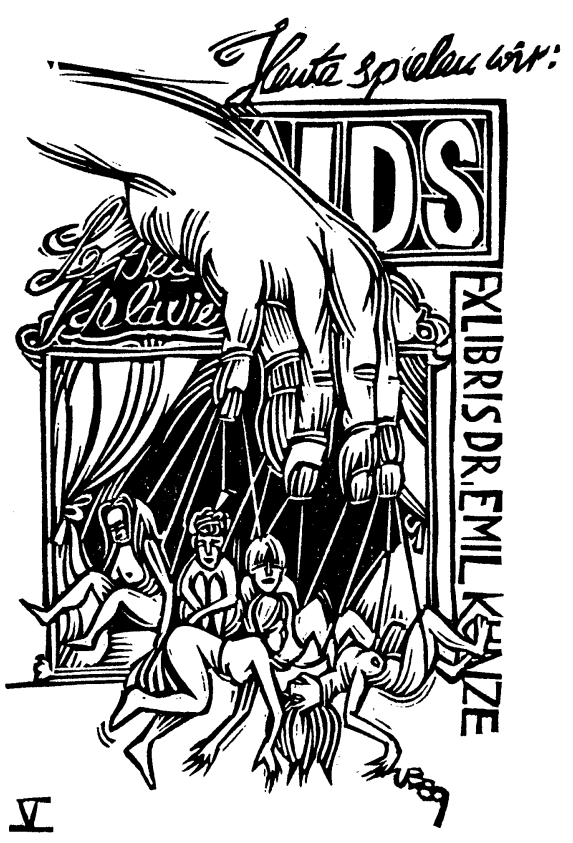

Abb. 17

Für Lutz Kowalzick, der sich in Hamburg habilitiert hat und jetzt Chefarzt der Hautklinik am Vogtland-Klinikum Plauen ist, hat Bernd Hieke eine sehr gefällige, fachbezogene Radierung (Abb. 18) mit einem schuppigen „Äskulap-Drachen“, einer Abbildung verschiedener Schuppenmuster und einer jungen Frau bei der Haarpflege gefertigt.

Die Pflege von Haaren, Nägeln und Haut ist auch Thema eines handkolorierten Kupferstichs von Oskar Roland Schroth (Abb. 19) für den Autor dieser Abhandlung. 


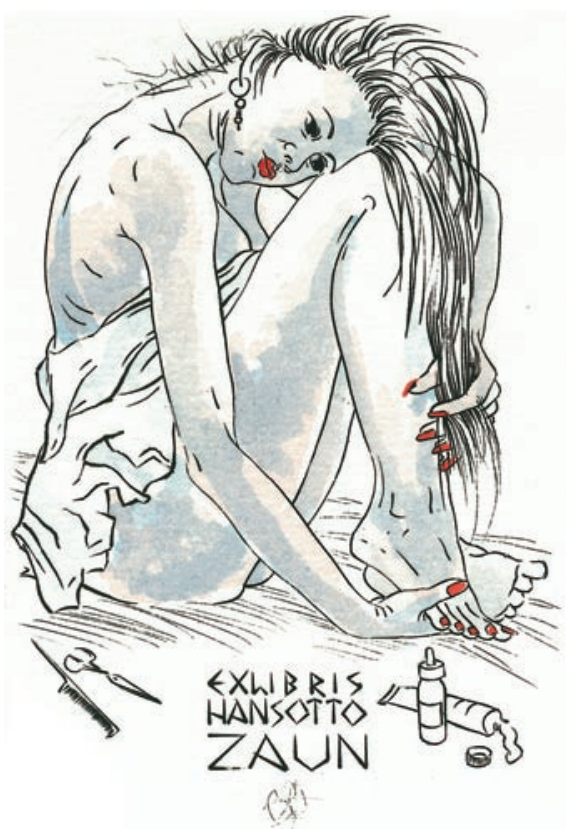

Ein weiteres der fünfzehn für mich gefertigten Exlibris ist abgebildet in der Monografie von Scholz [10], die meisten sind bisher nur in meinen Büchern zu finden.
${ }^{1}$ Benkel U. Zehn Exlibris zum Thema AIDS für Dr. med. Emil Kunze. Deggendorf: Verlag Utz Benkel (Kunstmappe), 1989

2 Blum G. Exlibris für Ärzte vom 16.Jahrhundert bis zur Gegenwart. Bonn: Hartmannbund-Verlag, 1983

${ }^{3}$ Ehring F. persönl. Mitteilung

${ }^{4}$ Happle R. An early drawing of Blaschko's lines. Brit J Dermatol 1993; 128: 464

5 Jung EG. persönl. Mitteilung

${ }^{6}$ Kreyenberg G. Exlibris für Ärzte. Köln: Dt. Ärzte-Verlag, 1983

${ }^{7}$ Kunze E. AIDS - Ein Nachwort (Beilage zu [1])

${ }^{8}$ Schirren C. Max Klinger - Carl Schirren. Briefwechsel 1910-1920. Hamburg: Dr. R. Krämer, 1988

${ }^{9}$ Scholz A. Der Gottlose borgt und gibt nicht wieder - Dermatologenexlibris um 1900. In: Herzberg J, Korting GW. Zur Geschichte der Deutschen Dermatologie. Berlin: Grosse, 1987: $101-107$

${ }^{10}$ Scholz A. Exlibris für Hautärzte. Leipzig: Thieme, 1990

${ }^{11}$ Scholz A. Exlibris berühmter Dermatologen. Hautarzt 1990; 41: $178-181$

12 Scholz A. Die Entdeckung von Exlibris bekannter Dermatologen. Akt Dermatol 2000; 26: $371-373$

${ }^{13}$ Scholz A, Ehring F, Zaun H. Exlibris berühmter Dermatologen. Berlin: 1995

14 Tormin A. Womit Ärzte in ihren Büchern Zeichen setzen. Medical Trib 2000; 35: 18

${ }^{15}$ Waehmer K. Bücherzeichen deutscher Ärzte. Leipzig: Kentaur-Verlag, 1919

${ }^{16}$ Wolff HH. persönl. Mitteilung

17 Zaun H. Ärztliches Handeln auf Exlibris. Med Report 1999; 23: 2

${ }^{18}$ Zaun H. Darstellungsweisen ärztlichen Handelns auf Exlibris. Dia-Klinik (Essex-Pharma GmbH). Homburg:128. Tagung der Vereinigung südwestdt. Dermatologen: 4-7

\section{Bebilderte Allergiegeschichte}

K.-C. Bergmann, I. Bergmann, H. Schadewaldt

Deisenhofen: Dustri-Verlag, 2004. 124 S. Kart. € 40,-.

ISBN 3-87185-341-0

Eine reich bebilderte Geschichte der Allergologie wird in einen kleinen, handlichen Band von 124 Seiten vorgelegt. Eine Symbiose der Allergologie und der Medizingeschichte vom Altertum über das Mittelalter in die Neuzeit. Sie basiert auf dem 4-bändigen Werk von H. Schadewaldt über die Geschichte der Allergologie, welches im selben Verlag vor gut 20 Jahren herausgekommen ist.

Kurze Texte und eindrückliche Abbildungen von Personen, Karten, epochalen Erkenntnissen werden in Erinnerung gerufen, und auf deren kulturelle Bedeutung wird hingewiesen. Auch die Reflexion in Wissenschaft, Kultur und im Gesundheitswesen wird angesprochen, resp. im Bild dargestellt. Es macht Freude zu blättern und zu verweilen, es erinnert und beglückt gleichermaßen. Es lohnt sich!

E. G. Jung, Heidelberg

\section{Fortschritte der Dermato-Allergologie}

\section{S. N. Jaffer, A. A. Qureshi}

C. J. Le Coz, G. Jelen, J.-P. Lepoittevin. Progrés en Dermato-Allergologie. Strasbourg, Paris: John Libbey Eurotext, 2003. 280 S. ISBN 2-7420-0493-9

Schriftlich liegt der Bericht des 24. Fortbildungskurses in Straßburg aus dem Jahre 2003 vor, 280 Seiten, gegliedert in 26 Kapitel. Hauptthema ist das allergische Kontaktekzem, seine Histologie (Erinnerungen an früher werden geweckt), die alten und neuen Testungen in der Wertung des Straßburger Altmeisters Edouard Grosshans, die verschiedenen Testreihen und Standards, neue Allergene (Haptene), neue berufliche Erkrankungen und auch die orale Provokation als Baboon-Snydrom. Forschung und Praxis durchdringen einander und Literatur ergänzt die Bemühung. Auch die Photodermatologie ist angesprochen und die inhalative Provokation mit ihren speziellen Krankheitsbildern. Eine reiche Ernte, welche dem scheidenden Klinikdirektor Prof. E. Grosshans in Dankbarkeit gewidmet wird. Eine bleibende Erinnerung und würdige Gabe.

E. G. Jung, Heidelberg 